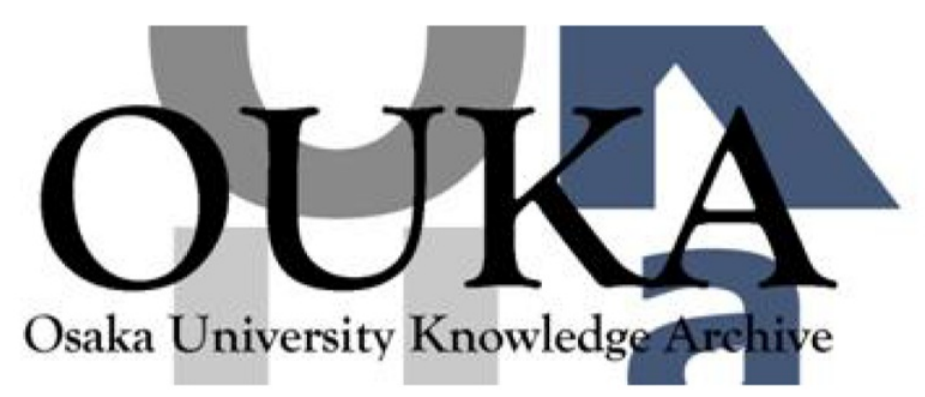

\begin{tabular}{|c|l|}
\hline Title & $\begin{array}{l}\text { Nearly diffraction-Limited line focusing of a } \\
\text { hard-X-ray beam with an elliptically figured } \\
\text { mirror }\end{array}$ \\
\hline Author(s) & $\begin{array}{l}\text { Yamauchi, Kazuto; Yamamura, Kazuya; Mimura, } \\
\text { Hidekazu et al. }\end{array}$ \\
\hline Citation & $\begin{array}{l}\text { Journal of Synchrotron Radiation. 9(5) p. 313- } \\
\text { p.316 }\end{array}$ \\
\hline Issue Date & $2002-09$ \\
\hline oaire:version & VoR \\
\hline URL & https://hdl.handle. net/11094/85462 \\
\hline rights & ○ International Union of Crystallography \\
\hline Note & \\
\hline
\end{tabular}

Osaka University Knowledge Archive : OUKA

https://ir. Library. osaka-u. ac. jp/

Osaka University 
JOURNAL OF

SYNCHROTRON

RADIATION

ISSN: 1600-5775

journals.iucr.org/s

\title{
Nearly diffraction-limited line focusing of a hard-X-ray beam with an elliptically figured mirror
}

\author{
Kazuto Yamauchi, Kazuya Yamamura, Hidekazu Mimura, Yasuhisa Sano, \\ Akira Saito, Alexei Souvorov, Makina Yabashi, Kenji Tamasaku, Tetsuya \\ Ishikawa and Yuzo Mori
}

J. Synchrotron Rad. (2002). 9, 313-316

\section{IIUCr Journals CRYSTALLOGRAPHY JOURNALS ONLINE}

Copyright (C) International Union of Crystallography

Author(s) of this article may load this reprint on their own web site or institutional repository provided that this cover page is retained. Republication of this article or its storage in electronic databases other than as specified above is not permitted without prior permission in writing from the IUCr.

For further information see https://journals.iucr.org/services/authorrights.html 


\section{Nearly diffraction-limited line focusing of a hard-X-ray beam with an elliptically figured mirror}

\author{
Kazuto Yamauchi, ${ }^{\text {a* }}$ Kazuya Yamamura, ${ }^{b}$ Hidekazu \\ Mimura, ${ }^{a}$ Yasuhisa Sano, ${ }^{a}$ Akira Saito, ${ }^{a}$ Alexei \\ Souvorov, ${ }^{c}$ Makina Yabashi, ${ }^{c}$ Kenji Tamasaku, ${ }^{d}$ \\ Tetsuya Ishikawa ${ }^{c, d}$ and Yuzo Mori ${ }^{a, b}$
}

${ }^{a}$ Department of Precision Science, Osaka University, YamadaOka 2-1, Suita, Osaka 565-0871, Japan, ${ }^{b}$ Research Center of Ultraprecision Science and Technology, Osaka University, Yamada-Oka 2-1, Suita, Osaka 565-0871, Japan, ' SPring-8/ Japan Synchrotron Radiation Research Institute (JASRI), Kouto 1-1-1, Mikazuki, Hyogo 679-5198, Japan, and ${ }^{d}$ SPring-8/RIKEN, Kouto 1-1-1, Mikazuki, Hyogo 679-5148, Japan.

E-mail: yamauchi@prec.eng.osaka-u.ac.jp

An elliptical mirror for X-ray microfocusing was manufactured using the new fabrication methods of elastic emission machining and plasma chemical vaporization machining. Surface profiles measured using stitching interferometry showed a maximum deviation around the ideal figure of $7 \mathrm{~nm}$ peak-to-valley. The mirror showed nearly diffraction-limited focusing performance, with a $200 \mathrm{~nm}$ line width at the focus. Wave-optical calculations, taking the measured surface profile into consideration, reproduced well the measured focusing properties both at and around the beam waist.

Keywords: elastic emission machining; plasma chemical vaporization machining; coherent X-rays; submicrometre focusing; elliptical mirrors.

\section{Introduction}

The inherently non-dispersive nature of totally external mirrors for $\mathrm{X}$-ray microfocusing is most attractive when we consider spectroscopic applications that usually require broad bandpass or energy tunability. For this purpose, Kirkpatrick-Baez (K-B) mirrors (Kirkpatrick \& Baez, 1948) are being developed at many synchrotron radiation facilities. This classical method utilizes two concave mirrors at a glancing angle to collect and focus X-rays in both the vertical and horizontal axes for X-ray microfocusing. However, microfocus mirrors for X-rays demand submicroradian figure perfection, as well as a surface roughness of less than $\sim 0.3 \mathrm{~nm}$ r.m.s. (Freund et al., 1990; Susini, 1995). Until quite recently, such an X-ray-quality mirror was only possible for spherical or flat figures, but not the elliptical figures required for $\mathrm{K}-\mathrm{B}$ mirrors. Benders have been developed to make elliptical figures from flat ones (Padmore et al., 1996; Yang et al., 1995; Howell et al., 2000; Hignette et al., 2001), and a differential deposition technique has been used to modify the figure of cylindrical substrates (Ice et al., 2000). The best focal size achieved using bent elliptical mirrors is $0.6 \times 0.2 \mu \mathrm{m}$ (Hignette et al., 2001), and using the differential deposition technique (Ice et al., 2000) is $0.4 \times 0.5 \mu \mathrm{m}$. Conventional polishing has also been attempted for manufacturing elliptical mirrors (Hayakawa et al., 2001), but the resulting slope error was of several microradians. By making the focal distance shorter to reduce the effect of slope error, a focus size of $2 \times 4 \mu \mathrm{m}$ was achieved. The observed focus sizes of the previous works are always larger than the design values. Convolution of the slope error with the designed focus size usually gives a good estimate of the observed size.
In this paper, we will show that aspherical X-ray-quality mirrors can be manufactured using recently developed machining methods (Mori, Yamamura \& Sano, 2000; Mori, Yamauchi et al., 2000; Yamauchi, Mimura et al., 2002). Measurement of the surface figure profile down to the $0.1 \mathrm{~mm}$ spatial frequency range enables waveoptical calculation of the focusing properties, predicting not only the focus size but also the focus profile and even the profiles in out-offocal planes. We have carried out line focusing tests of an elliptical mirror at the $1 \mathrm{~km}$ beamline at SPring-8 (Ishikawa et al., 2001), where we can expect coherent illumination. The observed line-focus profile, with $200 \mathrm{~nm}$ FWHM, agreed well with the wave-optically calculated profile.

\section{Figuring and figure testing methods}

We used the fabrication methods of elastic emission machining (EEM) (Mori et al., 1987, 1988, 2001) and plasma chemical vaporization machining (CVM) (Mori, Yamamura \& Sano, 2000; Mori, Yamauchi et al., 2000) as ultra-precise figuring and polishing methods to prepare both flat and figured X-ray mirrors. EEM is a chemical machining method utilizing reactivity of the surfaces of ultrafine powder particles (Mori et al., 1987, 1988). Numerically controlled EEM can create atomically flat and crystallographically nondamaged surfaces with a figuring of $0.1 \mathrm{~nm}$ range r.m.s. accuracy (Mori et al., 1988, 2001), although the removal rate is low compared with other figuring techniques. Plasma CVM, developed as a precise and effective preprocess of EEM, is a chemical-plasma process with an r.m.s. figure accuracy close to $1 \mathrm{~nm}$, having a high removal rate comparable with conventional grinding. A combined process of plasma CVM and EEM in series enables manufacture, in a reasonable time, of the ultra-precise X-ray optical components that are required for the highly coherent X-rays from the new generation of synchrotron sources.

One of the great advantages of numerically controlled EEM is its ability to correct surface figures using figure metrology data. However, the widely used long trace profiler (LTP) (Takacs, 1987) has insufficient spatial resolution $(\sim 1 \mathrm{~mm})$ for the correction to suppress interference fringes observed in the reflected beam (Mori et al., 2001; Yamauchi, Yamamura et al., 2002). Accordingly, we adopt a stitching interferometry (Bray, 1999) combining a microscopic phase-shifting interferometer with a large-area Fizeau's interferometer. In the stitching procedure, we use two parameters for optimization. One is the difference between the conventionally stitched profile and that measured directly with a large-area Fizeau's interferometer. The other is the superposition error between neighboring images of a microscopic interferometer. The two parameters are optimized with a weight factor related to the spatial frequency. As a result, we can evaluate the mirror surface profile with a slope accuracy at the $0.1 \mu \mathrm{rad}$ level over the spatial range from $0.1 \mathrm{~mm}$ to more than $100 \mathrm{~mm}$. Numerical calculation using the Fresnel-Kirchhoff integral (Born \& Wolf, 1997) reproduces the observed beam profiles reflected by flat mirrors fairly well when we use the surface profile measured with the stitching method as the boundary condition (Mori et al., 2001; Yamauchi, Yamamura et al., 2002).

\section{Elliptical mirror for $\mathbf{1 0 0} \mathrm{nm}$ range line-focusing}

The above figuring methods were applied to the manufacture of an elliptical mirror, with the parameters shown in Table 1, for linefocusing of coherent X-rays. The substrate material was an $\mathrm{Si}(111)$ single-crystal plate. To reflect up to $20 \mathrm{keV} \mathrm{X}$-rays, the maximum glancing angle was designed to be $1.55 \mathrm{mrad}$. A relatively long focal 
Table 1

Parameters of the manufactured mirror.

\begin{tabular}{ll}
\hline Substrate material & CZ-(111)Si single crystal \\
Surface coating & None \\
Effective mirror size in longitudinal direction & $90 \mathrm{~mm}$ \\
Length of ellipse & $500.15 \mathrm{~m}$ \\
Breadth of ellipse & $24.25 \mathrm{~mm}$ \\
Focus length & $300 \mathrm{~mm}$ \\
Glancing angle on optical axis & $1.40 \mathrm{mrad}$ \\
Maximum glancing angle & $1.55 \mathrm{mrad}$ \\
Acceptance width & $130 \mu \mathrm{m}$ \\
\hline
\end{tabular}

length $(300 \mathrm{~mm})$ was chosen to allow a sufficient working distance. To show the characteristic focusing at a photon energy of $15 \mathrm{keV}$, the numerically calculated result using the Fresnel-Kirchhoff integral for an ideal elliptical mirror is depicted in Fig. 1. The incident X-rays used for the calculation had the beam parameters obtained at the $1 \mathrm{~km}$-long beamline (BL29XUL) (Ishikawa et al., 2001) at SPring-8. The incident vertical slit of width $100 \mu \mathrm{m}$ was located at $1 \mathrm{~km}$ upstream of the mirror, where the transverse coherence length was assumed to be $50 \mu \mathrm{m}$. The FWHM and the width between the two first minima were found to be $200 \mathrm{~nm}$ and $400 \mathrm{~nm}$, respectively. As seen from the intensity ratio $(\sim 0.05)$ of the central and the first satellite peaks, full coherent illumination is realized all over the mirror surface (Born \& Wolf, 1997). In other words, diffractionlimited operation is in principle achievable in our setup.

Fig. 2 shows the measured surface figure and figure error of the manufactured elliptical mirror. The elliptical shape was first figured with plasma CVM in the spatial wavelength range above $5 \mathrm{~mm}$. Figure correction, with higher submillimeter spatial resolution, was then performed using numerically controlled EEM. The measured figure error around the ideal profile was less than $7 \mathrm{~nm}$ peak-tovalley.

\section{Focusing properties and wave-optical characterization}

The focusing test was performed at the $1 \mathrm{~km}$ beamline (BL29XUL) of SPring-8. Monochromatic X-rays at $15 \mathrm{keV}$ were prepared with the cryogenically cooled double-crystal $\mathrm{Si}(111)$ monochromator (Mochizuki et al., 2001) and guided to an experimental hutch of the beamline located $1 \mathrm{~km}$ from the center of the light-source undulator (Kitamura, 1998). The manufactured mirror was mounted on one of the goniometers (KOHZU, KTG-15) of the high-precision diffractometer system (Tamasaku et al., 2001; Ishikawa et al., 1992) installed in the experimental hutch. A wire-scanning method using a gold wire

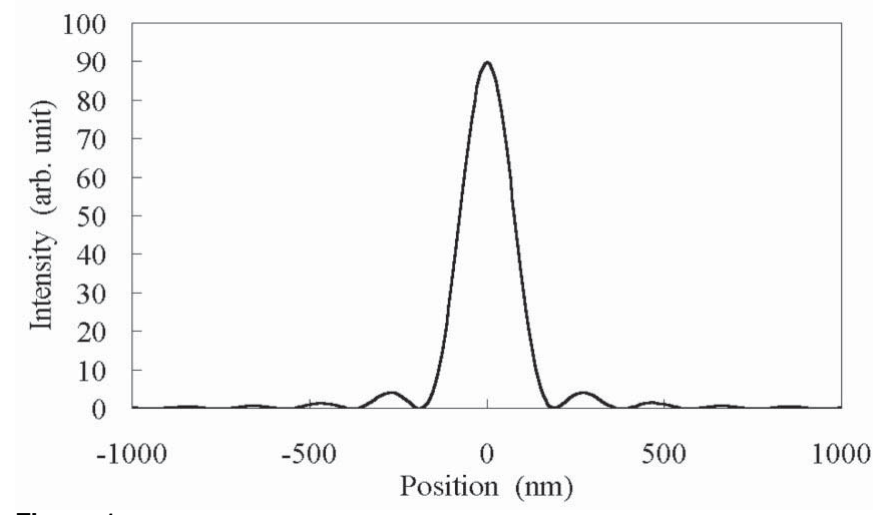

Figure 1

Focused beam profile calculated from ideal elliptical shape by using the Fresnel-Kirchhoff integral. of diameter $200 \mu \mathrm{m}$ placed in the focal plane was employed to measure the focused beam profile. A piezo-actuated translation stage (PI, P-733) enabled wire scanning with a $25 \mathrm{~nm}$ finest step. An avalanche photodiode detector (Kishimoto, 1992; Baron \& Ruby, 1994) placed behind the wire was used for measuring the beam intensity.

Fig. 3 shows the intensity profile measured under the best focus condition achieved and its differential curve corresponding to the focal beam profile, together with a calculated beam profile of the Fresnel-Kirchhoff integral using the measured mirror figure as the boundary condition, in which an evanescent wave is not taken into account. The half-value width of the focal line is as narrow as $200 \mathrm{~nm}$. Fairly good agreement between the measured and calculated profiles indicates that the present mirror-figure metrology is sufficient to predict focusing performance. This, in turn, shows that we must use wave-optical analysis even for hard-X-ray microfocusing by reflective mirror optics, instead of the conventional ray-tracing approach. The measured profile is completely raw data without any compensation to

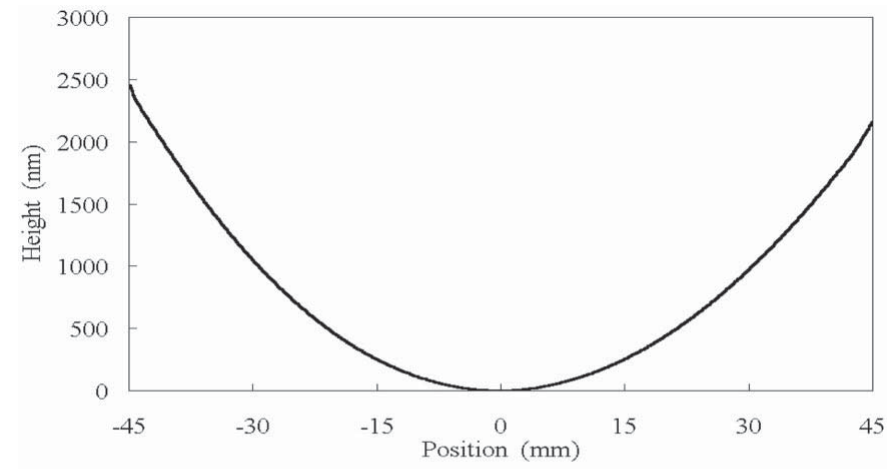

(a)

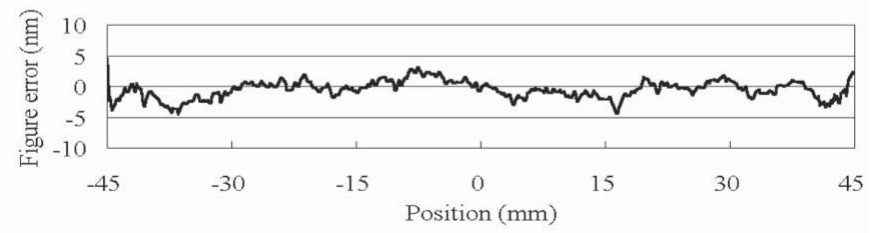

Figure 2

(b)

Figure $(a)$ and figure error $(b)$ of the manufactured elliptical mirror surface.

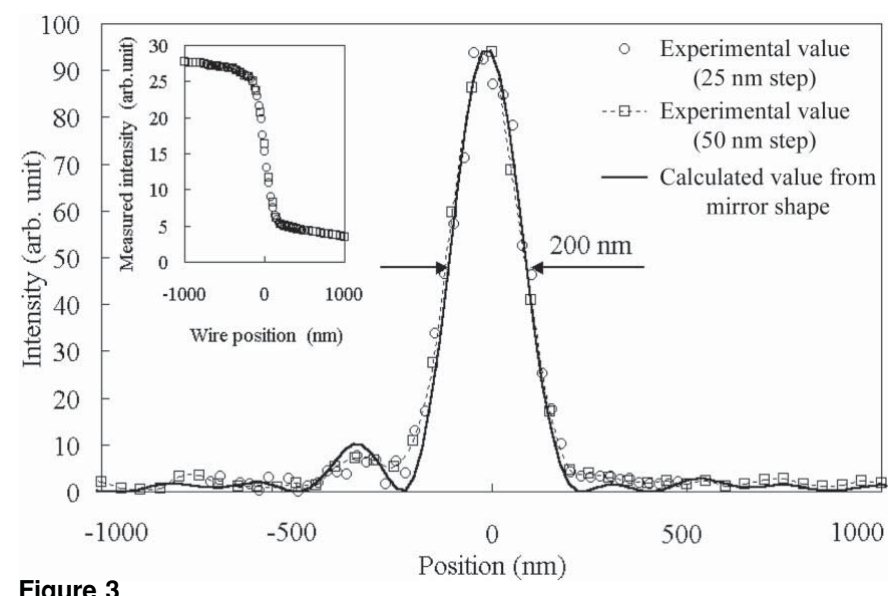

Figure 3

Observed and calculated beam profile at the focus point. The measured surface profile of the manufactured mirror is taken into account in the calculation. 
research papers

take the transmission effect at the gold-wire edge into account, because the transitionlayer width of the gold wire, which has a diameter of $200 \mu \mathrm{m}$, is as small as $10 \mathrm{~nm}$ and is smaller than the step in wire scanning. A small tail appearing at the left side of the main peak profile seems to originate in an edge effect of the gold wire. A relatively large shoulder at the left side of the center peak mainly originates in the residual figure error lying near the center of the manufactured mirror surface, which is seen to have the form of a sinusoid-like curve having a period of about $40 \mathrm{~mm}$ and a peakto-valley height of about $5 \mathrm{~nm}$. This kind of asymmetric figure error makes the beam profile asymmetric around the satellitepeak region and does not affect the main peak width as long as the height error is sufficiently smaller than $\lambda / \theta_{0}$, where $\lambda$ is the wavelength of the X-ray and $\theta_{0}$ is the glancing angle. The irradiance efficiency, defined by the ratio of the reflected photon flux to the incident one, was measured to be more than $80 \%$. The irradiance gain, defined by the ratio of the reflected photon flux density to the incident one, is estimated to be $\sim 400$, since the acceptance width of the present mirror is about $130 \mu \mathrm{m}$.

The wave-optical property of the present mirror is more clearly seen when we measure the beam profiles out of the focal plane. Fig. 4(a) shows the cross-sectional intensity profiles measured every $1 \mathrm{~mm}$ in the beam direction over the $\sim 5 \mathrm{~mm}$ range from the focal plane, while the corresponding calculated profiles for the ideal elliptical mirror are shown in Fig. 4(b). Constructive and destructive interference observed near the beam waist, which agree fairly well with the calculation, indicate that the present focusing is nearly diffraction limited.

\section{Discussion}

As illustrated earlier, the combination of CVM and EEM can be used to produce aspherical X-ray-quality mirrors for X-ray microfocusing. Although the data shown here were taken for an elliptical mirror with a figure error of $7 \mathrm{~nm}$ peak-to-valley from the ideal shape, we can reduce the error down to $\sim 1 \mathrm{~nm}$ from peak-to-valley by correcting the figure based on the interferometric metrology. Currently, the minimum achievable figure error is not limited by the EEM process but by the accuracy of the stitching interferometry. Therefore, further development of the figure metrology would enable us to manufacture aspherical mirrors with a figure error of less than $1 \mathrm{~nm}$ peak-to-valley.

The implications of the CVM and EEM methods go beyond aspherical mirrors. Since these machining methods produce no crystallographic damage, they are applicable to precise figuring of Bragg-diffraction optical elements such as X-ray inclined lenses (Hrdý, 1998). With coherent X-ray illumination, partly realized in the present third-generation synchrotron radiation sources and inevitable in the fourth-generation ones, even a flat-crystal monochromator could require an ultra-precise surface figure to maintain spatial homogeneity of the reflected beam or preserve coherence. The present machining methods and metrology would be good candidates for preparing optical elements, for both total external reflection and Bragg reflection, for fourth-generation synchrotron radiation sources.

The irradiance gain, defined by the ratio of the flux density of the incident beam to that at the focus, is $\sim 400$ for the present linefocusing mirror. When we use two elliptical mirrors that have the surface quality and figure error achieved here in the K-B arrangement for focusing in two dimensions, we can expect an irradiation gain of $\sim 160000$.

\section{Conclusion}

We have manufactured an X-ray-quality elliptical mirror with CVM and EEM. We achieved a $7 \mathrm{~nm}$ peak-to-valley figure error around the ideal elliptical shape over a $90 \mathrm{~mm}$ length on the mirror surface with $0.1 \mathrm{~mm}$ positional resolution. A line-focusing test carried out at the $1 \mathrm{~km}$ beamline of SPring- 8 gave a diffraction-limited focal line with $200 \mathrm{~nm}$ FWHM. Wave-optical calculations, taking the measured profile into consideration, reproduced well the measured focusing properties not only at the beam waist but also around the beam waist. Since the present figuring methods do not introduce any crystal damage, they are applicable for the ultra-smooth figuring of Braggdiffraction optical elements.

We would like to thank Alfred Q. R. Baron for his critical reading of the manuscript. Support for this research was provided by a Grantin-Aid for COE Research (No. 08CE2004) from the Ministry of Education, Sports, Culture, Science and Technology, Japan. 


\section{References}

Baron, A. Q. R. \& Ruby, S. L. (1994). Nucl. Instrum. Methods, A343, 517-526. Born, M. \& Wolf, E. (1997). Principles of Optics, 6th ed. Cambridge University Press.

Bray, M. (1999). Proc. SPIE, 3739, 259-273.

Freund, A. K., De Bergevin, F., Marot, G., Riekel, C., Susini, J., Lin, Z. \& Ziegler, E. (1990). Opt. Eng. 29, 928-941.

Hayakawa, S., Ikuta, N., Suzuki, M., Wakatsuki, M. \& Hirokawa, T. (2001). J. Synchrotron Rad. 8, 328-330.

Hignette, O., Rostaing, G., Cloetens, P., Rommeveaux, A., Ludwig, W. \& Freund, A. K. (2001). Proc. SPIE, 4499, 105-116.

Howell, M. R., Cambie, D., Duarte, R. M., Irick, S., MacDowell, A. A., Padmore, H. A., Renner, T. R., Rah, S. \& Sandler, R. (2000). Opt. Eng. 39, 2748-2762.

Hrdý, J. (1998). J. Synchrotron Rad. 5, 1206-1210.

Ice, G. E., Chung, J.-S., Tischler, J. Z., Lunt, A. \& Assoufid, L. (2000). Rev. Sci. Instrum. 71, 2635-2639.

Ishikawa, T., Tamasaku, K., Yabashi, M., Goto, S., Tanaka, Y., Yamazaki, H., Takeshita, K., Kimura, H., Ohashi, H., Matsushita, T. \& Ohata, T. (2001). Proc. SPIE, 4154, 1-10.

Ishikawa, T., Yoda, Y., Izumi, K., Suzuki, C. K., Zhang, X. W., Ando, M. \& Kikuta, S. (1992). Rev. Sci. Instrum. 63, 1015-1018.

Kirkpatrick, P. \& Baez, A. V. (1948). J. Opt. Soc. Am. 38, 766-774.

Kishimoto, S. (1992). Rev. Sci. Instrum. 63, 824-827.
Kitamura, H. (1998). J. Synchrotron Rad. 5, 184-188.

Mochizuki, T., Kohmura, Y., Awaji, A., Suzuki, Y., Baron, A., Tamasaku, K., Yabashi, M., Yamazaki, H. \& Ishikawa, T. (2001). Nucl. Instrum. Methods, A467/468, 647-649.

Mori, Y., Yamauchi, K. \& Endo, K. (1987). Precis. Eng. 9, 123-128.

Mori, Y., Yamauchi, K. \& Endo, K. (1988). Precis. Eng. 10, 24-28.

Mori, Y., Yamamura, K. \& Sano, Y. (2000). Rev. Sci. Instrum. 71, 4620-4626.

Mori, Y., Yamauchi, K., Yamamura, K., Mimura, H., Saito, A., Kishimoto, H., Sekito, Y., Kanaoka, M., Souvorov, A., Yabashi, M., Tamasaku, K. \& Ishikawa, T. (2001). Proc. SPIE, 4501, 30-42.

Mori, Y., Yamauchi, K., Yamamura, K. \& Sano, Y. (2000). Rev. Sci. Instrum. 71, 4627-4632.

Padmore, H. A., Howell, M. R., Irick, M. R., Renner, S., Sandler, T. \& Koo, Y.-M. (1996). Proc. SPIE, 2856, 145-156.

Susini, J. (1995). Opt. Eng. 34, 361-376.

Takacs, P. Z. (1987). Proc. SPIE, 749, 59-71.

Tamasaku, K., Tanaka, Y., Yabashi, M., Yamazaki, H., Kawamura, N., Suzuki, M. \& Ishikawa, T. (2001). Nucl. Instrum. Methods, A467/468, 686-689.

Yamauchi, K., Mimura, H., Inagaki, K. \& Mori, Y. (2002). Rev. Sci. Instrum. Submitted.

Yamauchi, K., Yamamura, K., Mimura, H., Saito, A., Kanaoka, M., Souvorov, A., Yabashi, M., Tamasaku, K., Ishikawa, T. \& Mori, Y. (2002). In preparation.

Yang, B. X., Rivers, M., Schildkamp, W. \& Eng, P. J. (1995). Rev. Sci. Instrum. 66, 2278-2280. 